\title{
The Neutral Stochastic Integrodifferential Equations with Jumps
}

\author{
Diem Dang Huan \\ Faculty of Basic Sciences, Bacgiang Agriculture and Forestry University, Bacgiang 21000, Vietnam
}

Correspondence should be addressed to Diem Dang Huan; diemdanghuan80@gmail.com

Received 2 April 2016; Accepted 28 June 2016

Academic Editor: Maria Bruzón

Copyright (C) 2016 Diem Dang Huan. This is an open access article distributed under the Creative Commons Attribution License, which permits unrestricted use, distribution, and reproduction in any medium, provided the original work is properly cited.

We study the existence and uniqueness of mild solutions for neutral stochastic integrodifferential equations with Poisson jumps under global and local Carathéodory conditions on the coefficients by means of the successive approximation. Furthermore, we give the continuous dependence of solutions on the initial value. Finally, an example is provided to illustrate the effectiveness of the obtained results.

\section{Introduction}

Stochastic evolution equations (SEEs) are well known to model problems from many areas of science and engineering, wherein quite often the future state of such systems depends not only on the present state but also on its past history (delay) leading to stochastic functional differential equations and it has played an important role in many ways such as the model of the systems in physics, chemistry, biology, economics, and finance from various points of the view (see, e.g., $[1,2]$ ).

Recently, SEEs in infinite dimensional spaces have been extensively studied by many authors (see, e.g., [3, 4] and the references therein). There is much interest in studying qualitative properties: existence and uniqueness, stability, invariant measure, and so forth for SEEs with Wiener process (see, e.g., $[3,5,6])$. Particularly, the existence and stability results of solution to SEEs and integrodifferential systems have also been considered in the literature (see, e.g., $[7,8]$ ). Furthermore, the problem of the existence and uniqueness of solution for neutral stochastic partial functional differential equation in the case where the coefficients do not satisfy the global Lipschitz condition was investigated by Cao et al. [9], Bao and Hou [10], and recently Govindan [11] and Diop et al. [12].

On the other hand, there have not been many studies of SEEs driven by jumps processes while these have begun to gain attention recently. To be more precise, Röckner and Zhang [13] showed by successive approximations the existence, uniqueness, and large deviation principle of SEEs with jumps. Luo and Taniguchi [14] considered the existence and uniqueness of mild solutions to SEEs with finite delay and Poisson jumps by the Banach fixed point theorem. For SEEs with jumps one can see recent monograph [15] as well as papers $([9,13,14,16]$ and the references therein). Motivated by the previously mentioned problems, we will extend some such results for the following neutral stochastic integrodifferential equations with Poisson jumps:

$$
\begin{aligned}
& d\left[x(t)-\Gamma\left(t, x_{t}\right)\right] \\
& =A\left[x(t)-\Gamma\left(t, x_{t}\right)\right] d t \\
& \quad+\left[\int_{0}^{t} K(t-s)\left[x(s)-\Gamma\left(s, x_{s}\right)\right] d s+F\left(t, x_{t}\right)\right] d t \\
& \quad+\Sigma\left(t, x_{t}\right) d W(t)+\int_{\mathcal{U}} L\left(t, x_{t}, v\right) \widetilde{N}(d t, d v), \\
& x(t)=\varphi(t), \quad-r \leq t \leq 0, r>0, T],
\end{aligned}
$$

with an initial function $x(t)=\varphi=\{\varphi(t):-r \leq t \leq 0\} \in$ $\mathscr{C}_{\mathscr{F}_{0}}^{b}([-r, 0] ; \mathbb{W})$; that is, $\varphi$ is an $\mathscr{F}_{0}$-measurable, $\mathscr{C}([-r, 0] ; \llbracket)$ value random variable such that $\mathbf{E}\|\varphi\|_{\mathscr{C}}^{2}<\infty$, and $A: D(A) \subset$ $\mathbb{U} \rightarrow \mathbb{U}, K(t): D(K(t)) \subset \mathbb{U} \rightarrow \mathbb{U}$ are linear, closed, and densely defined operators in a Hilbert space $\mathbb{H} ; x_{t}(\theta)=x(t+$ $\theta)$ for $\theta \in[-r, 0]$. Let the functions $F: \mathbb{R}^{+} \times \mathscr{C} \rightarrow \mathbb{H}, \Sigma:$

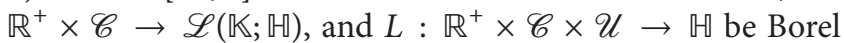
measurable and let $\Gamma: \mathbb{R}^{+} \times \mathscr{C} \rightarrow \mathbb{W}$ be continuous. 
The aim of our paper is to establish existence, uniqueness, and stability results for mild solution of (1) under global and local Carathéodory conditions in the Hilbert space based on successive approximation method. Our main results concerning (1) rely essentially on techniques using strongly continuous family of operators $\{R(t), t \geq 0\}$, defined on the Hilbert space $\mathbb{M}$ and called their resolvent (for the precise definition we can refer to Grimmer [17]).

The rest of this paper is organized as follows: In Section 2, we will give some necessary notations, concepts, and basic results about the Wiener process, Poisson jumps process, and deterministic integrodifferential equations. Section 3 is devoted to prove the existence and uniqueness of the solution. In Section 4, we study stability through the continuous dependence on the initial values. An example is given in Section 5 to illustrate the theory.

\section{Preliminaries Results}

This section is concerned with some basic concepts, notations, definitions, lemmas, and preliminary facts which are used through this paper. For more details on this section, we refer the reader to $[3,17-19]$.

Let $\left(\Omega, \mathscr{F},\left(\mathscr{F}_{t}\right)_{t \geqslant 0}, \mathbf{P}\right)$ be a complete probability space equipped with some filtration $\left(\mathscr{F}_{t}\right)_{t \geqslant 0}$ satisfying the usual conditions (i.e., it is right continuous and $\mathscr{F}_{0}$ contains all Pnull sets). Let $\left(\mathbb{Z},\|\cdot\|_{\mathbb{R}},\langle\cdot, \cdot\rangle\right)$ and $\left(\mathbb{K},\|\cdot\|_{\mathbb{K}},\langle\cdot, \cdot\rangle\right)$ denote two real separable Hilbert spaces, with their vectors norms and their inner products, respectively. We denote by $\mathscr{L}(\mathbb{K} ; \mathbb{U})$ the set of all linear bounded operators from $\mathbb{K}$ into $\mathbb{U}$, which is equipped with the usual operator norm $\|\cdot\|$.

Let $r>0$ and $\mathscr{C}:=C([-r, 0] ; \mathbb{U})$ denotes the family of all right continuous functions with left-hand limits (càdlàg) from $[-r, 0]$ to $\mathbb{H}$. The space $\mathscr{C}$ is assumed to be equipped with the norm $\|\varsigma\|_{\mathscr{C}}:=\sup _{-r \leq t \leq 0}\|\varsigma(t)\|_{\mathbb{H}}, \varsigma(t) \in \mathscr{C}$.

We also assume that $\mathscr{C}_{\mathscr{F}_{0}}^{b}([-r, 0]$; $\mathbb{H})$ denotes the family of all almost surely bounded, $\mathscr{F}_{0}$-measurable, $\mathscr{C}([-r, 0]$; $\mathbb{U})$ valued random variables. Further, we consider the Banach space $\mathbf{B}_{T}$ of all $\mathbb{H}$-valued $\mathscr{F}_{t}$-adapted càdlàg process $x(t)$ defined on $[0, T], T>0$ with $x(t)=\varphi(t), t \in[-r, 0]$ such that

$$
\|x\|_{\mathbf{B}_{T}}^{2}:=\mathbb{E} \sup _{0 \leq t \leq T}\|x(t)\|_{\mathbb{q}}^{2}<\infty .
$$

Let $W(t)$ be a $\mathbb{K}$-valued $\left(\mathscr{F}_{t}\right)_{t \geqslant 0}$-Wiener process defined on the probability space $(\Omega, \mathscr{F}, \mathbf{P})$ with covariance operator $Q$, where $Q$ is a positive, self-adjoint, trace class operator on $\mathbb{K}$. Let $\mathscr{L}_{2}:=\mathscr{L}_{2}\left(Q^{1 / 2} \mathbb{K} ; \mathbb{W}\right)$ denote the space of all HilbertSchmidt operators from $Q^{1 / 2} \mathbb{K}$ into $\mathbb{W}$ with the inner product $\langle\psi, \phi\rangle_{\mathscr{L}_{2}}=\operatorname{tr}\left(\psi Q \phi^{*}\right)$.

Let $p=p(t), t \in D_{p}$ (the domain of $p(t)$ ), be a stationary $\mathscr{F}_{t}$-Poisson point process taking its value in a measurable space $(\mathcal{U}, \mathscr{B}(\mathcal{U}))$ with $\sigma$-finite intensity measure $\lambda(d v)$ by $N(d t, d v)$, the Poisson counting measure associated with $p$; that is, $N(t, \mathscr{U})=\sum_{s \in D_{p}, s \leq t} \mathbb{q}_{\mathcal{U}}(p(s))$ for any measurable set $\mathcal{U} \in \mathscr{B}(\mathbb{K}-\{0\})$, which denotes the Borel $\sigma$-field of $(\mathbb{K}-\{0\})$. Let $\widetilde{N}(d t, d v):=N(d t, d v)-\lambda(d v) d t$ be the compensated Poisson measure that is independent of $W(t)$. Denote by
$\mathscr{P}^{2}([0, T] \times \mathscr{U} ; \mathbb{H})$ the space of all predictable mappings $L$ : $[0, T] \times \mathscr{U} \rightarrow \mathbb{H}$ for which $\int_{0}^{t} \int_{\mathcal{U}} \mathbf{E}\|L(t, v)\|_{\mathbb{T}}^{2} \lambda(d v) d t<$ $\infty$. We may then define the $\mathbb{-}$-valued stochastic integral $\int_{0}^{t} \int_{\mathcal{U}} L(t, v) \widetilde{N}(d t, d v)$, which is a centered square-integrable martingale. For the construction of this kind of integral, we can refer to Peszat and Zabczyk [15].

Next, to be able to access existence, uniqueness, and stability of mild solutions for (1) we need to introduce partial integrodifferential equations and resolvent operators.

Let $X, Z$ be two Banach spaces such that $\|z\|_{Z}:=\|A z\|_{X}+$ $\|z\|_{X}$ for all $z \in Z ; A$ and $K(t)$ are closed linear operators on $X$ and satisfy the following assumptions:

(H1) The operator $A: D(A) \subseteq X \rightarrow X$ is the infinitesimal generator of a strongly continuous semigroup on $X$.

(H2) For all $t \geq 0, K(t): D(K(t)) \subseteq X \rightarrow X$ is a closed linear operator, $D(A) \subseteq D(K(t))$, and $K(t) \in \mathrm{B}(Z, X)$ are the set of all bounded linear operators from $Z$ into $X$. For any $z \in Z$, the map $t \rightarrow K(t) z$ is bounded, differentiable and the derivative $t \rightarrow d K(t) z / d t$ is bounded uniformly continuous on $\mathbb{R}^{+}$.

By Theorem 2.3 in [17], we can see that $(\mathbf{H} 1)$ and (H2) imply the integrodifferential abstract Cauchy problem

$$
\begin{aligned}
\frac{d x(t)}{d t}=A x(t)+\int_{0}^{t} K(t-s) x(s) d s, & \\
& x(0)=x_{0} \in X,
\end{aligned}
$$

has an associated resolvent operator of bounded linear operators $R(t), t \geq 0$, on $X$. Hence, we can give the mild solution for the integrodifferential equation

$$
\begin{aligned}
\frac{d x(t)}{d t}=A x(t)+\int_{0}^{t} K(t-s) x(s) d s+ & \kappa(t), \\
x(0)=x_{0} \in X, & \\
x(t)=R(t) x_{0}+\int_{0}^{t} R(t-s) \kappa(s) d s, & \forall t \geq 0,
\end{aligned}
$$

where $\kappa:[0,+\infty) \rightarrow X$ is a continuous function.

Let us give the definition of mild solution for (1).

Definition 1. A càdlàg stochastic process $x:[-r, T] \rightarrow \mathbb{H}$, $0<T<+\infty$, is called a mild solution of (1) on $[-r, T]$ if

(i) $x(t)$ is $\mathscr{F}_{t}$-adapted, for all $t \in[-r, T]$,

(ii) for arbitrary $t \in[0, T], \mathbf{P}\left\{\omega: \int_{0}^{T}\|x(s)\|_{\mathbb{\sharp}}^{2} d s<+\infty\right\}=$ 1 , and $x(t)$ satisfies the following integral equation:

$$
\begin{aligned}
x(t)= & R(t)[\varphi(0)-\Gamma(0, \varphi)]+\Gamma\left(t, x_{t}\right) \\
& +\int_{0}^{t} R(t-s) F\left(s, x_{s}\right) d s \\
& +\int_{0}^{t} R(t-s) \Sigma\left(s, x_{s}\right) d W(s) \\
& +\int_{0}^{t} \int_{\mathcal{U}} R(t-s) L\left(s, x_{s}, v\right) \widetilde{N}(d s, d v),
\end{aligned}
$$


(iii) $x(t)=\varphi(t),-r \leq t \leq 0$.

Throughout this paper, we always assume the following assumptions are satisfied.

(H3)

(i) The growth condition: there exists a nonnegative real valued function $\gamma:[0, T] \times \mathbb{R}^{+} \rightarrow \mathbb{R}^{+}$, $(t, u) \mapsto \gamma(t, u)$, which is locally integrable in $t \geq 0$ for any fixed $u \geq 0$ and is continuous monotone nondecreasing in $u$ for any fixed $t \epsilon$ $[0, T]$. Furthermore, for any fixed $s \in[0, T]$ and $x_{s} \in L^{2}(\Omega, \mathscr{C})$, the following inequality is satisfied:

$$
\begin{gathered}
\mathbf{E}\left(\left\|F\left(s, x_{s}\right)\right\|_{\mathbb{G}}^{2}+\left\|\Sigma\left(s, x_{s}\right)\right\|_{\mathscr{L}_{2}}^{2}\right. \\
\left.+\int_{\mathscr{U}}\left\|L\left(s, x_{s}, v\right)\right\|_{\mathbb{E}}^{2} \lambda(d v)\right) \\
\quad \leq \gamma\left(s,\left(\mathbf{E} \sup _{r \in[0, s]}\left\|x_{r}\right\|_{\mathscr{C}}^{2}\right)\right) .
\end{gathered}
$$

(ii) For arbitrary nonnegative numbers $\beta$ and $w_{0}$, the integral equation

$$
w(t)=w_{0}+\beta \int_{0}^{t} \gamma(s, w(s)) d s
$$

has a global solution on $[0, T]$.

(H4)

(i) The global condition: there exists a nonnegative real valued function $\gamma:[0, T] \times \mathbb{R}^{+} \rightarrow \mathbb{R}^{+}$, $(t, u) \mapsto \gamma(t, u)$, which is locally integrable in $t \geq 0$ for any fixed $u \geq 0$ and is continuous monotone nondecreasing in $u$ for any fixed $t \epsilon$ $[0, T]$. Furthermore $\gamma(t, 0) \equiv 0$ and for any fixed $s \in[0, T]$ and $x_{s}, z_{s} \in L^{2}(\Omega, \mathscr{C})$, the following inequality is satisfied:

$$
\begin{aligned}
& \mathbf{E}\left(\left\|F\left(s, x_{s}\right)-F\left(s, z_{s}\right)\right\|_{\mathbb{G}}^{2}+\left\|\Sigma\left(s, x_{s}\right)-\Sigma\left(s, z_{s}\right)\right\|_{\mathscr{L}_{2}}^{2}\right. \\
& \left.\quad+\int_{\mathscr{U}}\left\|L\left(s, x_{s}, v\right)-L\left(s, z_{s}, v\right)\right\|_{\mathbb{Q}}^{2} \lambda(d v)\right) \\
& \quad \leq \gamma\left(s, \mathbf{E}\left(\sup _{r \in[0, s]}\left\|x_{r}-z_{r}\right\|_{\mathscr{C}}^{2}\right)\right) .
\end{aligned}
$$

(ii) If there exists a nonnegative continuous function $y(t)$ satisfying $y(0)=0$ and

$$
y(t) \leq \beta \int_{0}^{t} \gamma(s, y(s)) d s, \quad \forall t \in[0, T],
$$

then $y(t) \equiv 0$ on $[0, T]$, where $\beta$ is a positive number.
(H5)

(i) The local condition: for any integer $c>0$ there exists a nonnegative real valued function $\gamma_{c}$ : $[0, T] \times \mathbb{R}^{+} \rightarrow \mathbb{R}^{+},(t, u) \mapsto \gamma_{c}(t, u)$, which is locally integrable in $t \geq 0$ for any fixed $u \geq 0$ and is continuous monotone nondecreasing in $u$ for any fixed $t \in[0, T]$. Furthermore $\gamma_{c}(t, 0) \equiv 0$ and for any fixed $s \in[0, T]$ and $x_{s}, z_{s} \in L^{2}(\Omega, \mathscr{C})$ with $\mathbf{E}\left(\sup _{r \in[0, s]}\left\|x_{r}\right\|_{\mathscr{C}}\right) \vee \mathbf{E}\left(\sup _{r \in[0, s]}\left\|z_{r}\right\|_{\mathscr{C}}\right) \leq c$, the following inequality is satisfied:

$$
\begin{aligned}
& \mathbf{E}\left(\left\|F\left(s, x_{s}\right)-F\left(s, z_{s}\right)\right\|_{\mathbb{G}}^{2}+\left\|\Sigma\left(s, x_{s}\right)-\Sigma\left(s, z_{s}\right)\right\|_{\mathscr{L}_{2}}^{2}\right. \\
& \left.\quad+\int_{\mathscr{U}}\left\|L\left(s, x_{s}, v\right)-L\left(s, z_{s}, v\right)\right\|_{\mathbb{G}}^{2} \lambda(d v)\right) \\
& \quad \leq \gamma_{c}\left(s, \mathbf{E}\left(\sup _{r \in[0, s]}\left\|x_{r}-z_{r}\right\|_{\mathscr{C}}^{2}\right)\right) .
\end{aligned}
$$

(ii) If there exists a nonnegative continuous function $y(t)$ satisfying $y(0)=0$ and

$$
y(t) \leq \beta \int_{0}^{t} \gamma_{c}(s, y(s)) d s, \quad \forall t \in[0, T],
$$

then $y(t) \equiv 0$ on $[0, T]$, where $\beta$ is a positive number.

(H6) The contractive mapping: the mapping $\Gamma(t, u)$ satisfies that there exists a positive $\delta \in(0,1)$ such that, for any $x, z \in \mathscr{C}$ and $t \geq 0$,

$$
\|\Gamma(t, x)-\Gamma(t, z)\|_{\mathbb{q}} \leq \delta\|x-z\|_{\mathscr{C}} .
$$

Remark 2. The function $\gamma(t, u):=\alpha(t) \phi(u), t \geq 0, u \in \mathbb{R}^{+}$, where $\alpha(t)$ is nonnegative and locally integrable and $\phi(t)$ is a concave, continuous function, satisfies Osgood's condition; that is, it is a nondecreasing function with $\phi(0)=0$ and $\phi(u)>0, \forall u>0$, such that $\int_{0^{+}}(1 / \phi(u)) d u=\infty$. Then we can show that the function $\gamma(t, u)$ satisfies assumption (H4)(ii) (cf. [6]).

To illustrate this remark, we give two examples which satisfy the conditions of $\phi$ in Remark 2 . Let $\varepsilon \in(0,1)$. Set

$$
\begin{aligned}
& \phi_{1}(u)= \begin{cases}u \log \left(\frac{1}{u}\right) & \text { if } 0 \leq u \leq \varepsilon, \\
\phi_{1}(\varepsilon)+\phi_{1}^{\prime}(\varepsilon-)(u-\varepsilon) & \text { if } u>\varepsilon,\end{cases} \\
& \phi_{2}(u)= \begin{cases}u \log \left(\frac{1}{u}\right) \log \log \left(\frac{1}{u}\right) & \text { if } 0 \leq u \leq \varepsilon, \\
\phi_{2}(\varepsilon)+\phi_{2}^{\prime}(\varepsilon-)(u-\varepsilon) & \text { if } u>\varepsilon,\end{cases}
\end{aligned}
$$

where $\varepsilon$ is sufficiently small and $\phi_{i}^{\prime}, i=1,2$, is the left derivative of $\phi_{i}, i=1,2$, at the point $\varepsilon$. Then $\phi_{1}$ and $\phi_{2}$ are both concave nondecreasing functions definition on $\mathbb{R}^{+}$satisfying $\int_{0^{+}}\left(1 / \phi_{i}(x)\right) d x=\infty, i=1,2$. 
Remark 3. (1) If there exists a positive constant $\sigma$, such that $\gamma(t, u)=\sigma u, u \in \mathbb{H}$, then assumption (H4)-(i) implies the Lipschitz condition.

(2) From assumption (H5), for any fixed integer $c>0$, there exists $\eta_{c}>0$ such that $\gamma_{c}(t, u)=\eta_{c} u, u \in \mathbb{H}$; then assumption (H5)-(i) implies the local Lipschitz condition.

We now remark that for the proof of our main results we need the following lemmas.

Lemma 4 (see [2]). For $x, y \in \mathbb{H}$ and $\epsilon \in(0,1)$, the following inequality is true:

$$
\|x\|_{\mathbb{\square}}^{2} \leq \frac{1}{1-\epsilon}\|x-y\|_{\mathbb{\square}}^{2}+\frac{1}{\epsilon}\|y\|_{\mathbb{G}}^{2} .
$$

Lemma 5 (see [3, Proposition 7.3]). Suppose that $\Phi(t), t \geq$ 0 , is $\mathscr{L}_{2}$-valued predictable process and let $W_{A}^{\Phi}=\int_{0}^{t} S(t-$ $s) \Phi(s) d W(s), t \in[0, T]$. Then for any arbitrary $p>2$ there exists a constant $C(p, T)>0$ such that

$$
\begin{aligned}
& \mathbf{E} \sup _{t \in[0, T]}\left\|W_{A}^{\Phi}\right\|_{\mathbb{⿴}}^{p} \\
& \quad \leq C(p, T) \sup _{t \in[0, T]}\|S(t)\|^{p} \mathbf{E} \int_{0}^{T}\|\Phi(s)\|^{p} d s .
\end{aligned}
$$

Moreover, if $\mathbf{E} \int_{0}^{T}\|\Phi(s)\|^{p} d s<+\infty$, then there exists a continuous version of the process $\left\{W_{A}^{\Phi}\right\}_{t \geq 0}$. If $(S(t))_{t \geq 0}$ is a contraction semigroup, then the above result is true for $p \geq 2$.
Lemma 6 (see [20, Proposition 1.3]). Let $\Phi(t): \mathbb{R}^{+} \times \Omega \times$ $\mathcal{U} \rightarrow \mathbb{W}$ be a predictable function satisfying $\int_{0}^{T} \int_{\mathscr{U}} \| \Phi(s$, $u) \|^{2} \lambda(d v) d s<+\infty$ for all $t \geq 0 \mathbf{P}$ almost surely. Let $Z(t)=$ $\int_{0}^{t} \int_{\mathcal{U}} S(t-s) \Phi(s, v) \widetilde{N}(d s, d v)$. If $(S(t))_{t \geq 0}$ is a contraction semigroup, then $\forall p \in(0,2]$ there exists a constant $C(p, T)>0$ such that

$$
\begin{aligned}
& \mathbf{E} \sup _{t \in[0, T]}\|Z(t)\|_{\mathbb{\square}}^{p} \\
& \quad \leq C(p, T) \mathbf{E}\left(\int_{0}^{T} \int_{\mathscr{U}}\|\Phi(s, u)\|^{2} \lambda(d v) d s\right)^{p / 2} .
\end{aligned}
$$

\section{Existence and Uniqueness of Solution}

In this section, we will investigate the existence and uniqueness of the mild solution to (1) under the non-Lipschitz condition and a weakened linear growth condition.

We introduce the successive approximations to (5) as follows:

$$
x^{0}(t)= \begin{cases}\varphi(t), & \text { for } t \in[-r, 0] \\ R(t) \varphi(0), & \text { for } t \in[0, T]\end{cases}
$$

and $x^{n}$ for $n \geq 1$ is defined by

$$
\begin{aligned}
& x^{n}(t) \\
& = \begin{cases}\varphi(t) & \text { for } t \in[-r, 0], \\
R(t)[\varphi(0)-\Gamma(0, \varphi)]+\Gamma\left(t, x_{t}^{n}\right)+\int_{0}^{t} R(t-s) F\left(s, x_{s}^{n-1}\right) d s+\int_{0}^{t} R(t-s) \Sigma\left(s, x_{s}^{n-1}\right) d W(s)+\int_{0}^{t} \int_{\mathcal{U}} R(t-s) L\left(s, x_{s}^{n-1}, v\right) \widetilde{N}(d s, d v), & \forall t \in[0, T] .\end{cases}
\end{aligned}
$$

Theorem 7. Assume the assumptions of (H1)-(H4) and (H6) hold. Then, there exists a unique mild solution to (1) in $\mathbf{B}_{T}$.

Proof. The proof is split into the following three steps.

Step 1. We claim that the sequence $\left\{x^{n}(t)\right\}_{n \geq 0}$ is bounded. Obviously, $x^{0}(t) \in \mathbf{B}_{T}$. Moreover, we easily show that $x^{n}(t) \epsilon$ $\mathbf{B}_{T}$, for $t \in[0, T]$ and $n=1,2, \ldots$ In fact, from (18), for $t \in[0, T]$, using the basic inequality $|a+b+c+d|^{2} \leq 4|a|^{2}+$ $4|b|^{2}+4|c|^{2}+4|d|^{2}$, we can get

$$
\begin{aligned}
& \mathbf{E} \sup _{s \in[0, t]}\left\|x^{n}(s)-\Gamma\left(s, x_{s}^{n}\right)\right\|_{\mathbb{G}}^{2} \leq 4 \mathbf{E} \\
& \quad \sup _{s \in[0, t]}\|R(s)[\varphi(0)-\Gamma(0, \varphi)]\|_{\mathbb{Q}}^{2}+4[\mathbf{E} \\
& \cdot \sup _{s \in[0, t]}\left\|\int_{0}^{s} R(s-r) F\left(r, x_{r}^{n-1}\right) d r\right\|_{\mathbb{I}}^{2}+\mathbf{E}
\end{aligned}
$$

$$
\begin{aligned}
& \cdot \sup _{s \in[0, t]}\left\|\int_{0}^{s} R(s-r) \Sigma\left(r, x_{r}^{n-1}\right) d W(r)\right\|_{\mathbb{H}}^{2}+\mathbf{E} \\
& \left.\cdot \sup _{s \in[0, t]}\left\|\int_{0}^{s} \int_{\mathscr{U}} R(s-r) L\left(r, x_{r}^{n-1}, v\right) \widetilde{N}(d r, d v)\right\|_{\mathbb{T}}^{2}\right] \\
& =: 4\left(I_{1}+I_{2}\right) .
\end{aligned}
$$

By assumption (H6), with $M:=\sup _{s \in[0, t]}\|R(s)\|_{\mathbb{\sharp}}^{2}$, it follows that

$$
I_{1} \leq M(1+\delta)^{2} \mathbf{E}\|\varphi\|_{\mathscr{C}}^{2}
$$

Note that $\mathbf{E}\left(\sup _{s \in[0, t]}\left\|x_{s}^{n-1}\right\|_{\mathscr{C}}^{2}\right) \leq \mathbf{E}\left(\sup _{s \in[0, t]}\left\|x^{n-1}(s)\right\|_{\mathbb{q}}^{2}\right)+$ $\mathbf{E}\|\varphi\|_{\mathscr{C}}^{2}$. 
By using the Hölder inequality and Lemmas 5 and 6 , then, associated with assumption (H3) for the term $I_{2}$, we obtain

$$
I_{2} \leq C_{1} \int_{0}^{t} \gamma\left[s, \mathbf{E}\left(\sup _{r \in[0, s]}\left\|x^{n-1}(r)\right\|_{\mathbb{\boxplus}}^{2}+\|\varphi\|_{\mathscr{C}}^{2}\right)\right] d s,
$$

where $C_{1}$ is a positive constant.

Hence, putting (20) and (21) into (19) yields

$$
\begin{aligned}
\mathbf{E s u p}_{s \in[0, t]} & \left\|x^{n}(s)-\Gamma\left(s, x_{s}^{n}\right)\right\|_{\mathbb{H}}^{2} \\
\leq & 4 M(1+\delta)^{2} \mathbf{E}\|\varphi\|_{\mathscr{C}}^{2} \\
& +4 C_{1} \int_{0}^{t} \gamma\left[s, \mathbf{E}\left(\sup _{r \in[0, s]}\left\|x^{n-1}(r)\right\|_{\boxplus}^{2}+\|\varphi\|_{\mathscr{C}}^{2}\right)\right] d s .
\end{aligned}
$$

While, by Lemma 4 , it follows that

$$
\begin{aligned}
& \mathbf{E}\left(\sup _{s \in[0, t]}\left\|x^{n}(s)\right\|_{\mathbb{H}}^{2}+\|\varphi\|_{\mathscr{C}}^{2}\right) \\
& \leq\left[\frac{4 M(1+\delta)^{2}}{(1-\delta)^{2}}+\frac{1}{1-\delta}\right] \mathbf{E}\|\varphi\|_{\mathscr{C}}^{2}+\frac{4 C_{1}}{(1-\delta)^{2}} \\
& \cdot \int_{0}^{t} \gamma\left[s, \mathbf{E}\left(\sup _{r \in[0, s]}\left\|x^{n-1}(r)\right\|_{\mathbb{H}}^{2}+\|\varphi\|_{\mathscr{C}}^{2}\right)\right] d s .
\end{aligned}
$$

From assumption (H3)-(ii) we show that there is a solution $u_{t}$ that satisfies

$$
u_{t}=C_{2} \mathbf{E}\|\varphi\|_{\mathscr{C}}^{2}+C_{3} \int_{0}^{t} \gamma\left(\omega, u_{\omega}\right) d \omega,
$$

where $C_{2}:=4 M(1+\delta)^{2} /(1-\delta)^{2}+1 /(1-\delta) ; C_{3}:=$ $4 C_{1} /(1-\delta)^{2}$.

On the other hand, since $\mathbf{E}\|\varphi\|_{\mathscr{C}}^{2}<\infty$, we deduce that

$$
\mathbf{E}\left(\sup _{s \in[0, t]}\left\|x^{n}(s)\right\|_{\mathbb{A}}^{2}\right) \leq u_{t} \leq u_{T}<\infty .
$$

Hence, $x^{n}(t) \in \mathbf{B}_{T}$, for $t \in[0, T]$ and $n=1,2, \ldots$ This proves the boundedness of $\left\{x^{n}(t)\right\}_{n \geq 0}$.

Step 2. We claim that the sequence $\left\{x^{n}(t)\right\}_{n \geq 0}$ is a Cauchy sequence in $\mathbf{B}_{T}$. For $m, n \geq 0$ and $t \in[0, T]$, from (18), (H4), and Step 1, we can show that there exists a positive constant $\mathrm{C}_{4}$ such that

$$
\begin{aligned}
& \mathbf{E} \sup _{s \in[0, t]}\left\|x^{n+1}(s)-\Gamma\left(s, x_{s}^{n+1}\right)-x^{m+1}(s)+\Gamma\left(s, x_{s}^{m+1}\right)\right\|_{\mathbb{H}}^{2} \leq 3 \mathbf{E} \\
& \cdot \sup _{s \in[0, t]}\left\|\int_{0}^{s} R(s-r)\left[F\left(r, x_{r}^{n}\right)-F\left(r, x_{r}^{m}\right)\right] d r\right\|_{\text {H }}^{2}+3 \mathrm{E} \\
& \cdot \sup _{s \in[0, t]}\left\|\int_{0}^{s} R(s-r)\left[\Sigma\left(r, x_{r}^{n}\right)-\Sigma\left(r, x_{r}^{m}\right)\right] d W(r)\right\|_{\mapsto}^{2}+3 \mathbf{E} \\
& \sup _{s \in[0, t]}\left\|\int_{0}^{s} \int_{\mathscr{U}} R(s-r)\left[L\left(r, x_{r}^{n}, v\right)-L\left(r, x_{r}^{m}, v\right)\right] \widetilde{N}(d r, d v)\right\|_{\text {H }}^{2} \\
& \leq C_{4} \int_{0}^{t} \gamma\left(s, \mathbf{E}\left(\sup _{r \in[0, s]}\left\|x^{n}(r)-x^{m}(r)\right\|_{\mathbb{H}}^{2}\right)\right) d s .
\end{aligned}
$$

Therefore applying Lemma 4 and assumption (H6) again, we obtain

$$
\begin{gathered}
\mathbf{E} \sup _{s \in[0, t]}\left\|x^{n+1}(s)-x^{m+1}(s)\right\|_{\mathbb{H}}^{2} \leq \frac{C_{4}}{(1-\delta)^{2}} \\
\cdot \int_{0}^{t} \gamma\left(s, \mathbf{E}\left(\sup _{r \in[0, s]}\left\|x^{n}(r)-x^{m}(r)\right\|_{\mathbb{H}}^{2}\right)\right) d s .
\end{gathered}
$$

Let

$$
y(t):=\lim _{n, m \rightarrow+\infty} \sup \left(\mathbf{E} \sup _{s \in[0, t]}\left\|x^{n}(s)-x^{m}(s)\right\|_{\mathbb{H}}^{2}\right) .
$$

From (25), condition (H4)-(ii), and the Fatou lemma, we have

$$
y(t) \leq C_{5} \int_{0}^{t} \gamma(s, y(s)) d s,
$$

where $C_{5}:=C_{4} /(1-\delta)^{2}$.

By condition (H4)-(ii) we get $y(t)=0$, which implies that

$$
\lim _{n, m \rightarrow+\infty}\left(\operatorname{Esup}_{s \in[0, t]}\left\|x^{n}(s)-x^{m}(s)\right\|_{\mathbb{H}}^{2}\right)=0 .
$$

This shows that sequence $\left\{x^{n}(t)\right\}_{n \geq 0}$ is Cauchy sequence in $\mathbf{B}_{T}$. Step 3. We claim the existence and uniqueness of the solution to (1).

Existence. By Step 2, we known that $\left\{x^{n}(t)\right\}_{n \geq 0}$ is a Cauchy sequence in $\mathbf{B}_{T}$; then the standard Borel-Cantelli lemma argument can be used to show that, as $n \rightarrow \infty, x^{n}(t) \rightarrow x(t)$ holds uniformly for $t \in[0, T]$. So, taking limits on both sides of (18) we obtain that $x(t)$ is a solution to (1). This shows the existence.

Uniqueness. Let both $x(t)$ and $z(t)$ be two mild solutions of (1) in $\mathbf{B}_{T}$; then by the same way as Step 2, we can show that there exists a positive constant $C_{6}$ such that

$$
\begin{aligned}
& \operatorname{Esup}_{s \in[0, t]}\|x(s)-z(s)\|_{\mathbb{H}}^{2} \\
& \quad \leq C_{6} \int_{0}^{t} \gamma\left(s, \mathbf{E}\left(\sup _{r \in[0, s]}\|x(r)-z(r)\|_{\mathbb{H}}^{2}\right)\right) d s .
\end{aligned}
$$

We can apply (H4)-(ii) again and infer that $\mathbf{E} \sup _{s \in[0, t]} \| x(s)$ $z(s) \|_{\mathbb{W}}^{2}=0$, which further implies $x(s) \equiv z(s)$ almost surely for any $0 \leq s \leq T$. This completes the proof of Theorem 7 .

Next, we present the existence and uniqueness of mild solutions for (1) with the local Carathéodory conditions.

Theorem 8. Assume the assumptions of $(\mathbf{H} 1)-(\mathbf{H} 3)$ and $(\mathbf{H} 5)$ and (H6) with $\delta \in(0,1 / 2)$ hold. Then, there exists a unique mild solution to (1) in $\mathbf{B}_{T}$. 
Proof. Let $c$ be a natural integer and let $\bar{T} \in(0, T)$. We define the sequence of the functions $\left\{F_{c}\right\},\left\{\Sigma_{c}\right\}$, and $\left\{L_{c}\right\}$ as follows:

$$
\begin{aligned}
& F_{c}\left(t, x_{t}\right) \\
& = \begin{cases}F\left(t, x_{t}\right) & \text { if } \mathbf{E}\left(\sup _{s \in[0, t]}\left\|x_{s}\right\|_{\mathscr{C}}\right) \leq c, \\
F\left(t, \frac{c x_{t}}{\mathbf{E}\left(\sup _{s \in[0, t]}\left\|x_{s}\right\|_{\mathscr{C}}\right)}\right) & \text { if } \mathbf{E}\left(\sup _{s \in[0, t]}\left\|x_{s}\right\|_{\mathscr{C}}\right)>c,\end{cases} \\
& \Sigma_{c}\left(t, x_{t}\right) \\
& = \begin{cases}\Sigma\left(t, x_{t}\right) & \text { if } \mathbf{E}\left(\sup _{s \in[0, t]}\left\|x_{s}\right\|_{\mathscr{C}}\right) \leq c, \\
\Sigma\left(t, \frac{c x_{t}}{\mathbf{E}\left(\sup _{s \in[0, t]}\left\|x_{s}\right\|_{\mathscr{C}}\right)}\right) & \text { if } \mathbf{E}\left(\sup _{s \in[0, t]}\left\|x_{s}\right\|_{\mathscr{C}}\right)>c,\end{cases} \\
& L_{c}\left(t, x_{t}, v\right) \\
& = \begin{cases}L\left(t, x_{t}, v\right) & \text { if } \mathbf{E}\left(\sup _{s \in[0, t]}\left\|x_{s}\right\|_{\mathscr{C}}\right) \leq c, \\
L\left(t, \frac{c x_{t}}{\mathbf{E}\left(\sup _{s \in[0, t]}\left\|x_{s}\right\|_{\mathscr{C}}\right)}, v\right) & \text { if } \mathbf{E}\left(\sup _{s \in[0, t]}\left\|x_{s}\right\|_{\mathscr{C}}\right)>c .\end{cases}
\end{aligned}
$$

Then, the functions $\left\{F_{c}\right\},\left\{\Sigma_{c}\right\}$, and $\left\{L_{c}\right\}$ satisfy assumption (H3) and the following inequality:

$$
\begin{aligned}
& \mathrm{E}\left(\left\|F_{c}\left(s, x_{s}\right)-F_{c}\left(s, z_{s}\right)\right\|_{\mathscr{H}}^{2}\right. \\
& \quad+\left\|\Sigma_{c}\left(s, x_{s}\right)-\Sigma_{c}\left(s, z_{s}\right)\right\|_{\mathscr{L}_{2}^{0}}^{2} \\
& \left.\quad+\int_{\mathcal{U}}\left\|L_{c}\left(s, x_{s}, v\right)-L_{c}\left(s, z_{s}, v\right)\right\|_{\mathscr{H}}^{2} \lambda(d v)\right) \\
& \quad \leq \gamma_{c}\left(s, \mathbf{E}\left(\sup _{r \in[0, s]}\left\|x_{r}-z_{r}\right\|_{\mathscr{C}}^{2}\right)\right),
\end{aligned}
$$

where $x_{s}, z_{s} \in L^{2}(\Omega, \mathscr{C}), s \in[0, \bar{T}]$.

Thus, by Theorem 7, there exists a unique solution $x^{c}(t) \epsilon$ $\mathbf{B}_{T}$ and $x^{c+1}(t) \in \mathbf{B}_{T}$, respectively, to the following equations:

$$
\begin{aligned}
x^{c}(t) & \\
= & R(t)[\varphi(0)-\Gamma(0, \varphi)]+\Gamma\left(t, x_{t}^{c}\right) \\
& +\int_{0}^{t} R(t-s) F_{c}\left(s, x_{s}^{c}\right) d s \\
& +\int_{0}^{t} R(t-s) \Sigma_{c}\left(s, x_{s}^{c}\right) d W(s) \\
& +\int_{0}^{t} \int_{\mathscr{U}} R(t-s) L_{c}\left(s, x_{s}^{c}, v\right) \widetilde{N}(d s, d v),
\end{aligned}
$$

$$
\begin{aligned}
x^{c+1}( & (t) \\
= & R(t)[\varphi(0)-\Gamma(0, \varphi)]+\Gamma\left(t, x_{t}^{c+1}\right) \\
& +\int_{0}^{t} R(t-s) F_{c+1}\left(s, x_{s}^{c+1}\right) d s \\
& +\int_{0}^{t} R(t-s) \Sigma_{c+1}\left(s, x_{s}^{c+1}\right) d W(s) \\
& +\int_{0}^{t} \int_{\mathscr{U}} R(t-s) L_{c+1}\left(s, x_{s}^{c+1}, v\right) \widetilde{N}(d s, d v) .
\end{aligned}
$$

Now define the stopping times

$$
\begin{aligned}
& \tau_{c}:=\bar{T} \wedge \inf \left\{t \in[0, T] \mid \mathbf{E}\left(\sup _{s \in[0, t]}\left\|x_{s}^{c}\right\|_{\mathscr{C}}\right) \geq c\right\}, \\
& \tau_{c+1} \\
& :=\bar{T} \\
& \wedge \inf \left\{t \in[0, T] \mid \mathbf{E}\left(\sup _{s \in[0, t]}\left\|x_{s}^{c+1}\right\|_{\mathscr{C}}\right) \geq c+1\right\}, \\
& \bar{\tau}_{c}:=\tau_{c} \wedge \tau_{c+1} .
\end{aligned}
$$

We claim that $x^{c+1}(t)=x^{c}(t)$, for all $t \in\left[0, \bar{T} \wedge \bar{\tau}_{c}\right]$, a.s. $\omega$. By (34) and for $s \in\left[0, \bar{\tau}_{c}\right]$,

$$
\begin{aligned}
& F_{c+1}\left(s, x_{s}^{c}\right)=F_{c}\left(s, x_{s}^{c}\right), \\
& \Sigma_{c+1}\left(s, x_{s}^{c}\right)=\Sigma_{c}\left(s, x_{s}^{c}\right), \\
& L_{c+1}\left(s, x_{s}^{c}\right)=L_{c}\left(s, x_{s}^{c}\right),
\end{aligned}
$$

and estimated as above we infer that there exist positive constants $C_{7}, C_{8}$ such that

$$
\begin{aligned}
& \mathbf{E} \sup _{s \in\left[0, t \wedge \bar{\tau}_{c}\right]}\left\|x^{c+1}(s)-x^{c}(s)\right\|_{\mathbb{H}}^{2} \leq 4 \delta^{2} \mathbf{E} \sup _{s \in\left[0, t \wedge \bar{\tau}_{c}\right]} \| x^{c+1}(s) \\
& -x^{c}(s)\left\|_{\mathbb{H}}^{2}+4 M T \int_{0}^{t \wedge \bar{\tau}_{c}}\right\| F_{c+1}\left(s, x_{s}^{c+1}\right) \\
& -F_{c+1}\left(s, x_{s}^{c}\right)\left\|_{\mathbb{H}}^{2} d s+C_{7} \int_{0}^{t \wedge \bar{\tau}_{c}}\right\| \Sigma_{c+1}\left(s, x_{s}^{c+1}\right) \\
& -\Sigma_{c+1}\left(s, x_{s}^{c}\right) \|_{\mathbb{H}}^{2} d s \\
& +C_{8} \int_{0}^{t \wedge \bar{\tau}_{c}} \int_{\mathscr{U}}\left\|L_{c+1}\left(s, x_{s}^{c+1}, v\right)-L_{c+1}\left(s, x_{s}^{c}, v\right)\right\|_{\mathbb{H}}^{2} \\
& \cdot \lambda(d v) d s .
\end{aligned}
$$


Hence, by assumption (H5)-(i) we have the following inequalities:

$$
\begin{aligned}
& \mathbf{E} \sup _{s \in[0, t]}\left\|x^{c+1}\left(s \wedge \bar{\tau}_{c}\right)-x^{c}\left(s \wedge \bar{\tau}_{c}\right)\right\|_{\mathbb{Q}}^{2} \leq \frac{4 M T}{1-4 \delta^{2}} \\
& \cdot \int_{0}^{t} \| F_{c+1}\left(s \wedge \bar{\tau}_{c}, x_{s \wedge \bar{\tau}_{c}}^{c+1}\right)-F_{c+1}(s \\
& \left.\wedge \bar{\tau}_{c}, x_{s \wedge \bar{\tau}_{c}}^{c}\right)\left\|_{\mathbb{\square}}^{2} d s+\frac{C_{7}}{1-4 \delta^{2}} \int_{0}^{t}\right\| \Sigma_{c+1}(s \\
& \left.\wedge \bar{\tau}_{c}, x_{s \wedge \bar{\tau}_{c}}^{c+1}\right)-\Sigma_{c+1}\left(s \wedge \bar{\tau}_{c}, x_{s \wedge \bar{\tau}_{c}}^{c}\right) \|_{\mathbb{Q}}^{2} d s \\
& +\frac{C_{8}}{1-4 \delta^{2}} \int_{0}^{t} \int_{\mathscr{U}} \| L_{c+1}\left(s \wedge \bar{\tau}_{c}, x_{s \wedge \bar{\tau}_{c}^{c}}^{c+1}, v\right) \\
& -L_{c+1}\left(s \wedge \bar{\tau}_{c}, x_{s \wedge \bar{\tau}_{c}}^{c}, v\right) \|_{\mathbb{Q}}^{2} \lambda(d v) d s \\
& \leq \frac{4 M T+C_{7}+C_{8}}{1-4 \delta^{2}} \int_{0}^{t} \gamma_{c+1}\left(s \wedge \bar{\tau}_{c},\right. \\
& \left.\mathbf{E}\left(\sup _{r \in[0, s]}\left\|x^{c+1}\left(r \wedge \bar{\tau}_{c}\right)-x^{c}\left(r \wedge \bar{\tau}_{c}\right)\right\|_{\mathbb{Q}}^{2}\right)\right) d s .
\end{aligned}
$$

For all $t \in[0, \bar{T}]$, by assumption (H5)-(ii) we obtain that

$$
\mathbf{E} \sup _{s \in[0, t]}\left\|x^{c+1}\left(s \wedge \bar{\tau}_{c}\right)-x^{c}\left(s \wedge \bar{\tau}_{c}\right)\right\|_{\mathbb{Q}}^{2}=0 .
$$

This means that, for all $t \in\left[0, \bar{T} \wedge \bar{\tau}_{c}\right]$, we always have

$$
x^{c+1}(t)=x^{c}(t), \quad \text { a.s. } \omega .
$$

For each $\omega \in \Omega$, there exists $c_{0}(\omega)>0$, such that $\bar{T} \epsilon$ $\left(0, \bar{\tau}_{c_{0}}\right]$. For all $t \in[0, \bar{T}]$, define $x(t)$ by

$$
x(t)=x^{c_{0}}(t) .
$$

Since $x\left(t \wedge \bar{\tau}_{c}\right)=x^{c}\left(t \wedge \bar{\tau}_{c}\right)$, it holds that

$$
\begin{aligned}
x\left(t \wedge \bar{\tau}_{c}\right) & \\
= & R(t)[\varphi(0)-\Gamma(0, \varphi)]+\Gamma\left(t, x_{t}^{c}\right) \\
& +\int_{0}^{t \wedge \bar{\tau}_{c}} R(t-s) F_{c}\left(s, x_{s}^{c}\right) d s \\
& +\int_{0}^{t \wedge \bar{\tau}_{c}} R(t-s) \Sigma_{c}\left(s, x_{s}^{c}\right) d W(s) \\
& +\int_{0}^{t \wedge \bar{\tau}_{c}} \int_{\mathcal{U}} R(t-s) L_{c}\left(s, x_{s}^{c}, v\right) \widetilde{N}(d s, d v)
\end{aligned}
$$

$$
\begin{aligned}
= & R(t)[\varphi(0)-\Gamma(0, \varphi)]+\Gamma\left(t, x_{t}\right) \\
& +\int_{0}^{t \wedge \bar{\tau}_{c}} R(t-s) F\left(s, x_{s}\right) d s \\
& +\int_{0}^{t \wedge \bar{\tau}_{c}} R(t-s) \Sigma\left(s, x_{s}\right) d W(s) \\
& +\int_{0}^{t \wedge \bar{\tau}_{c}} \int_{\mathcal{U}} R(t-s) L\left(s, x_{s}, v\right) \widetilde{N}(d s, d v) .
\end{aligned}
$$

Letting $c \rightarrow \infty$, for all $t \in[0, T]$, we infer that

$$
\begin{aligned}
x(t)= & R(t)[\varphi(0)-\Gamma(0, \varphi)]+\Gamma\left(t, x_{t}\right) \\
& +\int_{0}^{t} R(t-s) F\left(s, x_{s}\right) d s \\
& +\int_{0}^{t} R(t-s) \Sigma\left(s, x_{s}\right) d W(s) \\
& +\int_{0}^{t} \int_{\mathcal{U}} R(t-s) L\left(s, x_{s}, v\right) \widetilde{N}(d s, d v) .
\end{aligned}
$$

The uniqueness is obtained by stopping our process. The proof for Theorem 8 is thus complete.

\section{Stability of Solution}

In this section, we study the stability through the continuous dependence of mild solutions on the initial value. From now on, we will use $x^{\varphi}(t)$ to represent the mild solution of (1) to emphasize that the solution depends on the initial value $\varphi$. We need the following assumption:

(H7) For all $t \in[0, T], x, z \in \mathscr{C}$, there exists a positive constant $\Lambda$ such that

$$
\begin{aligned}
& \|F(t, x)-F(t, z)\|_{\mathbb{\square}}^{2}+\|\Sigma(t, x)-\Sigma(t, z)\|_{\mathscr{L}_{2}}^{2} \\
& \quad+\int_{\mathscr{U}}\|L(t, x, v)-L(t, z, v)\|_{\mathbb{a}}^{2} \lambda(d v) \\
& \leq \Lambda\|x-z\|_{\mathscr{C}}^{2} .
\end{aligned}
$$

Theorem 9. Let assumptions (H1), (H2), (H6) with $\delta \in$ $(0,1 / \sqrt{5})$, and $(\boldsymbol{H} 7)$ be satisfied. Then the mild solution of (1) is continuous in the initial value $\varphi$ (with respect to the strong topology on $\mathbb{H})$.

Proof. Let $x^{\varphi_{1}}(t)$ and $x^{\varphi_{2}}(t)$ be two mild solutions of (1) with initial values $\varphi_{1}$ and $\varphi_{2}$, respectively. Then, for all $t \in[0, T]$ we can show that there exists a positive constant $C_{9}$ such that

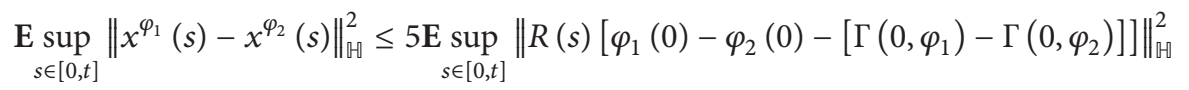

$$
\begin{aligned}
& +5 \mathbf{E} \sup _{s \in[0, t]}\left\|\Gamma\left(s, x_{s}^{\varphi_{1}}\right)-\Gamma\left(s, x_{s}^{\varphi_{2}}\right)\right\|_{\mathbb{H}}^{2}+5 \mathbf{E} \sup _{s \in[0, t]}\left\|\int_{0}^{s} R(s-r)\left[F\left(r, x_{r}^{\varphi_{1}}\right)-F\left(r, x_{r}^{\varphi_{2}}\right)\right] d r\right\|_{\mathbb{H}}^{2}
\end{aligned}
$$




$$
\begin{aligned}
& +5 \mathbf{E} \sup _{s \in[0, t]}\left\|\int_{0}^{s} R(s-r)\left[\Sigma\left(r, x_{r}^{\varphi_{1}}\right)-\Sigma\left(r, x_{r}^{\varphi_{2}}\right)\right] d W(r)\right\|_{\mathbb{H}}^{2} \\
& +5 \mathbf{E} \sup _{s \in[0, t]}\left\|\int_{0}^{s} \int_{\mathscr{U}} R(s-r)\left[L\left(r, x_{r}^{\varphi_{1}}, v\right)-L\left(r, x_{r}^{\varphi_{2}}, v\right)\right] \widetilde{N}(d r, d v)\right\|_{\mathbb{G}}^{2} \\
& \leq 10 M(1+\delta)^{2} \mathbf{E}\left\|\varphi_{1}-\varphi_{2}\right\|_{\mathscr{C}}^{2}+5 \delta^{2} \mathbf{E} \sup _{s \in[0, t]}\left\|x^{\varphi_{1}}(s)-x^{\varphi_{2}}(s)\right\|_{\mathbb{G}}^{2} \\
& +C_{9} \int_{0}^{t} \mathbf{E} \sup _{r \in[0, s]}\left\|x^{\varphi_{1}}(s)-x^{\varphi_{2}}(s)\right\|_{\mathbb{G}}^{2} d s .
\end{aligned}
$$

Thus,

$$
\begin{aligned}
\underset{s \in[0, t]}{\mathbf{E} \sup _{s}}\left\|x^{\varphi_{1}}(s)-x^{\varphi_{2}}(s)\right\|_{\mathbb{A}}^{2} \\
\leq \frac{10 M(1+\delta)^{2}}{1-5 \delta^{2}} \mathbf{E}\left\|\varphi_{1}-\varphi_{2}\right\|_{\mathscr{C}}^{2} \\
\quad+\frac{C_{9}}{1-5 \delta^{2}} \int_{0}^{t} \mathbf{E} \sup _{r \in[0, s]}\left\|x^{\varphi_{1}}(s)-x^{\varphi_{2}}(s)\right\|_{\mathbb{Q}-\mathbb{R}}^{2} d s .
\end{aligned}
$$

Applying Gronwall's inequality, we have

$$
\begin{aligned}
& \mathbf{E} \sup _{s \in[0, t]}\left\|x^{\varphi_{1}}(s)-x^{\varphi_{2}}(s)\right\|_{\mathbb{\square}}^{2} \\
& \quad \leq \frac{10 M(1+\delta)^{2}}{1-5 \delta^{2}} e^{\left(C_{9} /\left(1-5 \delta^{2}\right)\right) t} \mathbf{E}\left\|\varphi_{1}-\varphi_{2}\right\|_{\mathscr{C}}^{2},
\end{aligned}
$$

which means the mild solution is continuous in the initial value. This completes the proof of Theorem 9.

\section{Application}

In this section, an example is provided to illustrate the obtained theory. We consider the following neutral stochastic integrodifferential equations with Poisson jumps of the form:

$$
\begin{aligned}
& \frac{\partial}{\partial t}\left[u(t, \xi)-\int_{-r}^{0} \Upsilon(t, u(t+\theta, \xi)) d \theta\right] \\
& \quad=\frac{\partial^{2}}{\partial \xi^{2}}\left[u(t, \xi)-\int_{-r}^{0} \Upsilon(t, u(t+\theta, \xi)) d \theta\right] \\
& \quad+\int_{0}^{t} k(t-s) \frac{\partial^{2}}{\partial \xi^{2}}\left[u(s, \xi)-\int_{-r}^{0} \Upsilon(s, u(s+\theta, \xi)) d \theta\right] d s \\
& \quad+\int_{-r}^{0} f(t, u(t+\theta, \xi)) d \theta+\sigma(t, u(t+\theta, \xi)) d W(t)
\end{aligned}
$$

$$
\begin{gathered}
+\int_{u} u(t+\theta, \xi) v \widetilde{N}(d t, d v) \quad \text { for } t \geq 0, \xi \in[0, \pi], \\
u(t, 0)-\int_{-r}^{0} \Upsilon(t, u(t+\theta, 0)) d \theta=0 \text { for } t \geq 0, \\
u(t, \pi)-\int_{-r}^{0} \Upsilon(t, u(t+\theta, \pi)) d \theta=0 \quad \text { for } t \geq 0, \\
u(\theta, \xi)=u_{0}(\theta, \xi) \quad \text { for } \theta \in[-r, 0], \xi \in[0, \pi]
\end{gathered}
$$

where $W(t)$ is a standard one-dimensional Wiener process, $\mathcal{U}=\left\{v \in \mathbb{R}: 0<\|v\|_{\mathbb{R}} \leq a, a>0\right\} ; \Upsilon, f: \mathbb{R}^{+} \times \mathbb{R} \rightarrow \mathbb{R}$ and $\sigma: \mathbb{R}^{+} \times \mathbb{R} \rightarrow \mathscr{L}(\mathbb{R})$ are continuous function; $k: \mathbb{R}^{+} \rightarrow \mathbb{R}$ is continuous; and $u_{0}:[-r, 0] \times[0, \pi] \rightarrow \mathbb{R}$ is given càdlàg function such that $u_{0}(\cdot) \in L^{2}([0, \pi])$ is $\mathscr{F}_{0}$-measurable and satisfies $\mathbf{E}\left\|u_{0}\right\|_{\mathscr{C}}^{2}<\infty$.

Let $p=p(t), t \in D_{p}$, be a $\mathbb{K}$-valued $\sigma$-finite stationary Poisson point process (independent of $W(t)$ ) on a complete probability space with the usual condition $\left(\Omega, \mathscr{F},\left(\mathscr{F}_{t}\right)_{t \geqslant 0}, \mathbf{P}\right)$. Let $\widetilde{N}(d s, d v):=N(d s, d v)-\lambda(d v) d s$, with the characteristic measure $\lambda(d v)$ on $\mathscr{U} \in \mathscr{B}(\mathbb{K}-\{0\})$. Assume that $\int_{\mathscr{U}} v^{2} \lambda(d v)<$ $\infty$.

To rewrite (48) into the abstract form of (1) we consider the space $\mathbb{H}=L^{2}([0, \pi])$ with the norm $\|\cdot\|$. Let $e_{n}:=$ $\sqrt{2 / \pi} \sin n x, n=1,2,3, \ldots$, denote the completed orthonormal basics in $\mathbb{H}$ and $W(t)=\sum_{n=1}^{\infty} \sqrt{\lambda_{n}} \beta_{n}(t) e_{n}, t \geq 0, \lambda_{n}>$ 0 , where $\left\{\beta_{n}(t)\right\}_{n \geq 0}$ are one-dimensional standard Brownian motions mutually independent on a usual complete probability space $\left(\Omega, \mathscr{F},\left(\mathscr{F}_{t}\right)_{t \geqslant 0}, \mathbf{P}\right)$.

Defining $A: \mathbb{H} \rightarrow \mathbb{H}$ by $A=\partial^{2} / \partial x^{2}$, with domain $D(A)=$ $\mathbb{H}^{2}([0, \pi]) \cap \mathbb{T}_{0}^{1}([0, \pi])$, here $\mathbb{H}_{0}^{1}([0, \pi])=\left\{w \in L^{2}([0, \pi]):\right.$ $\left.\partial w / \partial z \in L^{2}([0, \pi]), w(0)=w(\pi)=0\right\}$, and $\mathbb{H}^{2}([0, \pi])=$ $\left\{w \in L^{2}([0, \pi]): \partial w / \partial z, \partial^{2} w / \partial z^{2} \in L^{2}([0, \pi])\right\}$. Then $A x=$ $-\sum_{n=1}^{\infty} n^{2}\left\langle x, e_{n}\right\rangle e_{n}, x \in D(A)$, where $n=1,2,3, \ldots$ is also the orthonormal set of eigenvector of $A$. It is well known that $A$ is the infinitesimal generator of a strongly continuous semigroup $\{S(t)\}_{t \geq 0}$ on $\mathbb{W}$ and is given (see Pazy [18, page 70]) by $S(t) x=\sum_{n=1}^{\infty} e^{-n^{2} t}\left\langle x, e_{n}\right\rangle e_{n}, x \in \mathbb{H}$. Thus, $(\mathbf{H} 1)$ is true.

Let $K(t): D(A) \subset \mathbb{T} \rightarrow \mathbb{W}$ be the operator defined by $K(t)(z)=k(t) A z$ for $t \geq 0$ and $z \in D(A)$. 
Now we assume the following.

(i) There exists a constant $C_{\Upsilon}, 0<r C_{\Upsilon} \sqrt{\pi}<1 / \sqrt{5}$, such that for $t \geq 0$ and $\nu_{1}, \nu_{2} \in \mathbb{R}$

$$
\left|\Upsilon\left(t, v_{1}\right)-\Upsilon\left(t, v_{2}\right)\right| \leq C_{\Upsilon}\left|v_{1}-v_{2}\right|
$$

(ii) There exists a constant $C_{f}, 0<r C_{f} \sqrt{\pi}<1$, such that for $t \geq 0$ and $v_{1}, v_{2} \in \mathbb{R}$

$$
\left|f\left(t, v_{1}\right)-f\left(t, v_{2}\right)\right|^{2} \leq C_{f} \gamma\left(t,\left|v_{1}-v_{2}\right|^{2}\right),
$$

(iii) For $t \geq 0$ and $v_{1}, v_{2} \in \mathbb{R}:\left|\sigma\left(t, v_{1}\right)-\sigma\left(t, v_{2}\right)\right|^{2} \leq$ $\gamma\left(t,\left|\nu_{1}-v_{2}\right|^{2}\right)$.

Let $\mathscr{C}=\mathscr{C}([-r, 0] ; \mathbb{H})$, for $\xi \in[0, \pi], \phi \in \mathscr{C}$ and define the operators $\Gamma, F: \mathbb{R}^{+} \times \mathscr{C} \rightarrow \mathbb{H}, \Sigma: \mathbb{R}^{+} \times \mathscr{C} \rightarrow \mathscr{L}(\mathbb{K} ; \mathbb{H})$, and $L: \mathbb{R}^{+} \times \mathscr{C} \times \mathcal{U} \rightarrow \mathbb{H}$ by

$$
\begin{aligned}
\Gamma(t, \phi)(\xi) & =\int_{-r}^{0} \Upsilon(t, \phi(\theta)(\xi)) d \theta, \\
F(t, \phi)(\xi) & =\int_{-r}^{0} f(t, \phi(\theta)(\xi)) d \theta, \\
\Sigma(t, \phi)(\xi) & =\sigma(t, \phi(\theta)(\xi)), \\
L(t, \phi(\xi), v) & =\phi(\xi) v .
\end{aligned}
$$

If we put

$$
\begin{aligned}
x(t) & =u(t, \xi), \quad \text { for } t \geq 0, \xi \in[0, \pi], \\
\varphi(\theta)(\xi) & =u_{0}(\theta, \xi), \quad \text { for } \theta \in[-r, 0], \xi \in[0, \pi],
\end{aligned}
$$

then (48) takes the following abstract form:

$$
\begin{aligned}
& d\left[x(t)-\Gamma\left(t, x_{t}\right)\right] \\
& =A\left[x(t)-\Gamma\left(t, x_{t}\right)\right] d t \\
& \quad+\left[\int_{0}^{t} K(t-s)\left[x(s)-\Gamma\left(s, x_{s}\right)\right] d s+F\left(t, x_{t}\right)\right] d t \\
& \quad+\Sigma\left(t, x_{t}\right) d W(t)+\int_{\mathcal{U}} L\left(t, x_{t}, v\right) \widetilde{N}(d t, d v), \\
& x(t)=\varphi(t), \quad-r \leq t \leq 0, r>0 .
\end{aligned}
$$

Moreover, if $k$ is bounded and $C^{1}$ function, where $C$ stand for the space of all continuous functions such that $k^{\prime}$ is bounded and uniformly continuous, then $(\mathbf{H} 1)$ and $(\mathbf{H} 2)$ are satisfied and hence there exists a resolvent operator $(R(t))_{t \geq 0}$ on $\mathbb{H}$. As a consequence of the continuity of $\Upsilon, f$, it follows that $\Gamma, F$ are continuous on $\mathbb{R}^{+} \times \mathscr{C}$ with values in $\mathbb{M}$, and from the continuity of $\sigma$ it follows that $\Sigma$ is continuous on $\mathbb{R}^{+} \times \mathscr{C}$ with values in $\mathscr{L}(\mathbb{K}, \mathbb{H})$. Thus, (48) can be expressed as (1) with $A, \Gamma, K, F, \Sigma$, and $L$ as defined above.

By assumption (i), we have $\left\|\Gamma\left(t, \phi_{1}\right)-\Gamma\left(t, \phi_{2}\right)\right\|_{L^{2}([0, \pi])} \leq$ $r C_{\Upsilon} \sqrt{\pi}\left\|\phi_{1}-\phi_{2}\right\|_{\mathscr{C}}$.
Further, by assumptions (ii) and (iii),

$$
\begin{aligned}
& \left\|F\left(t, \phi_{1}\right)-F\left(t, \phi_{2}\right)\right\|_{L^{2}([0, \pi])}^{2} \\
& \quad \leq r C_{f} \sqrt{\pi} \gamma\left(t,\left\|\phi_{1}-\phi_{2}\right\|_{\mathscr{C}}^{2}\right), \\
& \left\|\Sigma\left(t, \phi_{1}\right)-\Sigma\left(t, \phi_{2}\right)\right\|_{\mathscr{L}_{2}} \leq \gamma\left(t,\left\|\phi_{1}-\phi_{2}\right\|_{\mathscr{C}}^{2}\right) .
\end{aligned}
$$

On the other hand, in hypotheses (ii) and (iii) above, if there exists a positive constant $\rho$, such that $\gamma(t, u)=\rho u$, then there exists a positive constant $\Lambda$ such that assumption (H7) is established. Hence, all the assumptions of Theorems 7 and 9 are fulfilled. Therefore, there exists a unique mild solution of (48) by Theorem 7. Furthermore, this solution depends on the initial value by Theorem 9 .

\section{Competing Interests}

The author declares that there are no competing interests regarding the publication of this paper.

\section{References}

[1] W. Grecksch and C. Tudor, Stochastic Evolution Equations: A Hilbert Space Approach, Academi, Berlin, Germany, 1995.

[2] X. Mao, Stochastic Differential Equations and Applications, Horwood Publishing, Chichester, UK, 1997.

[3] G. Da Prato and J. Zabczyk, Stochastic Equations in Infinite Dimensions, vol. 44, Cambridge University Press, Cambridge, UK, 1992.

[4] A. Ichikawa, "Stability of semilinear stochastic evolution equations," Journal of Mathematical Analysis and Applications, vol. 90, no. 1, pp. 12-44, 1982.

[5] K. Liu, Stability of Infinite Dimensional Equations with Applications, Chapman \& Hall/CRC, London, UK, 2006.

[6] T. Taniguchi, "Successive approximations to solutions of stochastic differential equations," Journal of Differential Equations, vol. 96, no. 1, pp. 152-169, 1992.

[7] T. Caraballo, J. Real, and T. Taniguchi, "The exponential stability of neutral stochastic delay partial differential equations," Discrete and Continuous Dynamical Systems A, vol. 18, no. 2-3, pp. 295-313, 2007.

[8] G. Da Prato and M. Iannelli, "Existence and regularity for a class of integro-differential equations of parabolic type," Journal of Mathematical Analysis and Applications, vol. 112, no. 1, pp. 3655, 1985.

[9] G. Cao, K. He, and X. Zhang, "Successive approximations of infinite dimensional SDEs with jump," Stochastics and Dynamics, vol. 5, no. 4, pp. 609-619, 2005.

[10] J. Bao and Z. Hou, "Existence of mild solutions to stochastic neutral partial functional differential equations with nonLipschitz coefficients," Computers \& Mathematics with Applications, vol. 59, no. 1, pp. 207-214, 2010.

[11] T. E. Govindan, "Sample path exponential stability of stochastic neutral partial functional differential equations," Journal of Numerical Mathematics and Stochastics, vol. 3, no. 1, pp. 1-12, 2011.

[12] M. K. Diop, K. Enzzinbi, and M. Lo, "Mild solution of neutral stochastic partial functional integrodifferential equations with non-Lipschitz coefficients," Afrika Matematika, vol. 24, no. 4, pp. 671-682, 2013. 
[13] M. Röckner and T. Zhang, "Stochastic evolution equations of jump type: existence, uniqueness and large deviation principles," Potential Analysis, vol. 26, no. 3, pp. 255-279, 2007.

[14] J. Luo and T. Taniguchi, "The existence and uniqueness for nonLipschitz stochastic neutral delay evolution equations driven by Poisson jumps," Stochastics and Dynamics, vol. 9, no. 1, pp. 135152, 2009.

[15] S. Peszat and J. Zabczyk, Stochastic Partial Differential Equations with Lévy Noise, Cambridge University Press, Cambridge, UK, 2007.

[16] L. Bo, K. Shi, and Y. Wang, "Approximating solutions of neutral stochastic evolution equations with jumps," Science in China Series A: Mathematics, vol. 52, no. 5, pp. 895-907, 2009.

[17] R. C. Grimmer, "Resolvent operators for integral equations in a Banach space," Transactions of the American Mathematical Society, vol. 273, no. 1, pp. 333-349, 1982.

[18] A. Pazy, Semigroup of Linear operators and Applications to Partial Differential Equations, Springer, New York, NY, USA, 1992.

[19] S. Watanabe and N. Ikeda, Stochastic Differential Equations and Diffusion Processes, vol. 24 of North-Holland Mathematical Library, North-Holland, New York, NY, USA, 1st edition, 1981.

[20] E. Hausenblas and J. Seidler, "Stochastic convolutions driven by martingales: maximal inequalities and exponential integrability," Stochastic Analysis and Applications, vol. 26, no. 1, pp. 98119, 2008. 


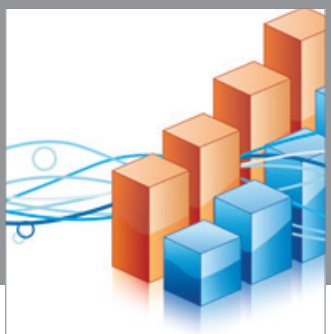

Advances in

Operations Research

vatem alat4

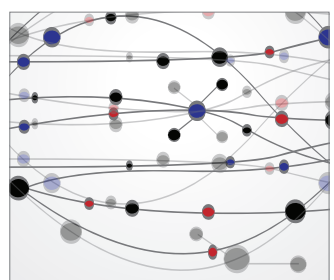

\section{The Scientific} World Journal
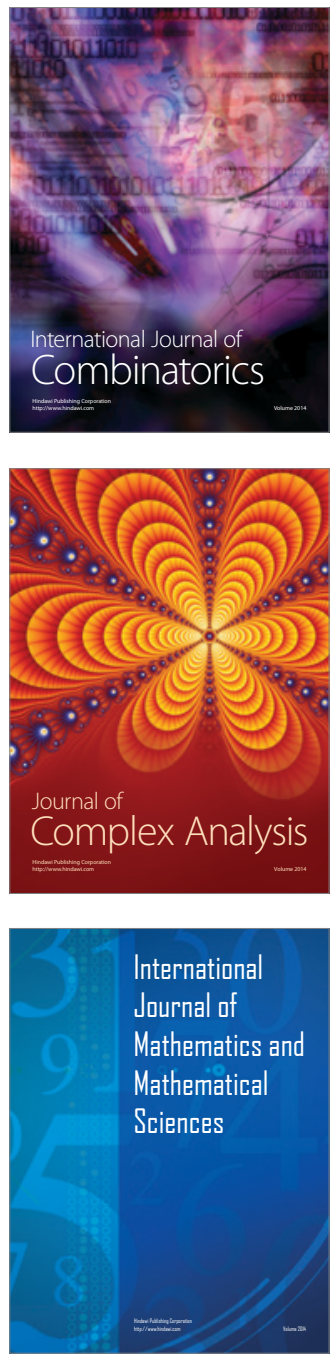
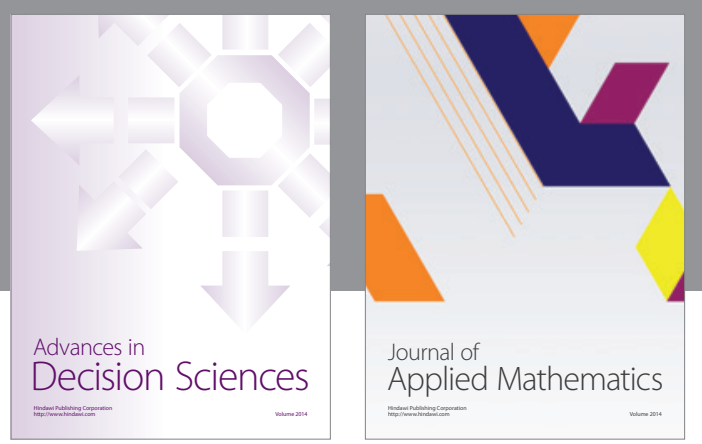

Algebra

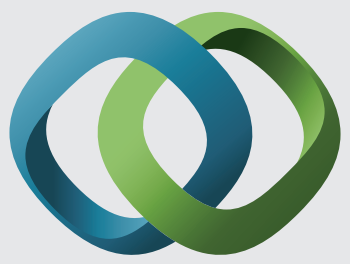

\section{Hindawi}

Submit your manuscripts at

http://www.hindawi.com
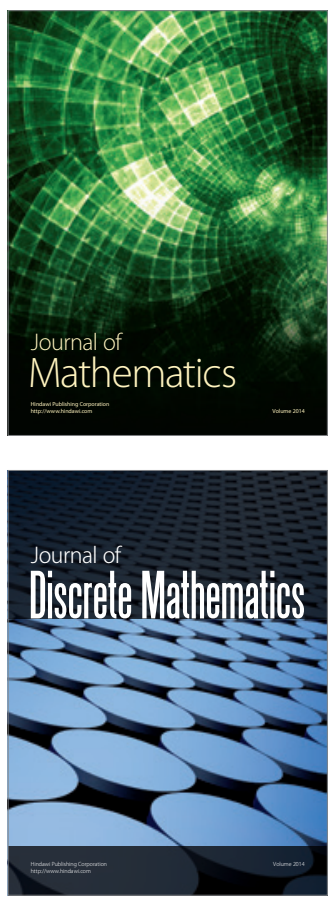

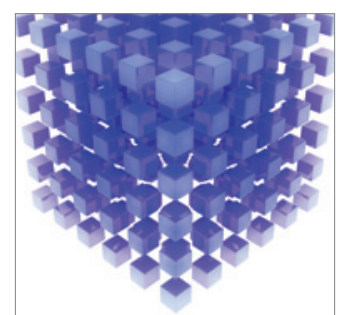

Mathematical Problems in Engineering
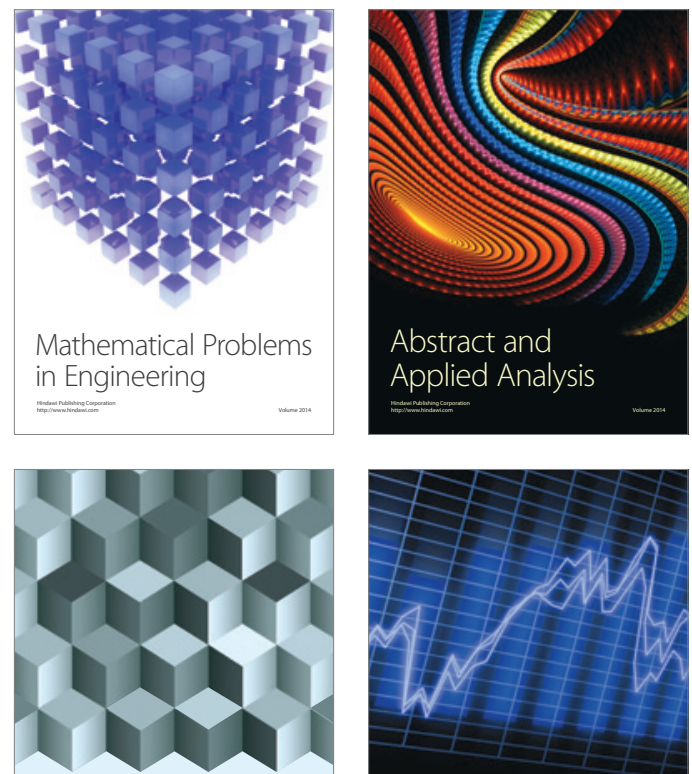

Journal of

Function Spaces

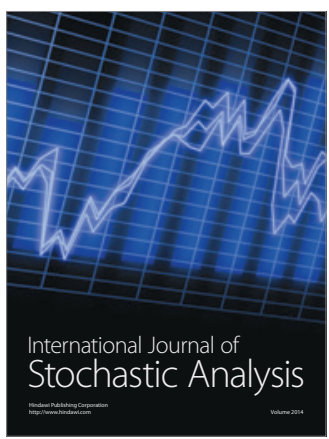

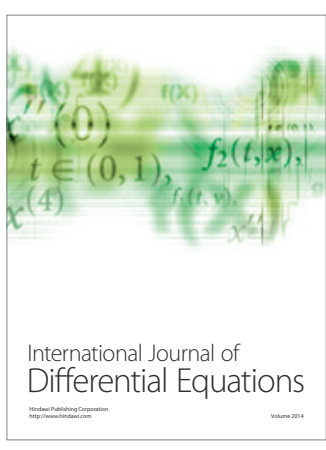
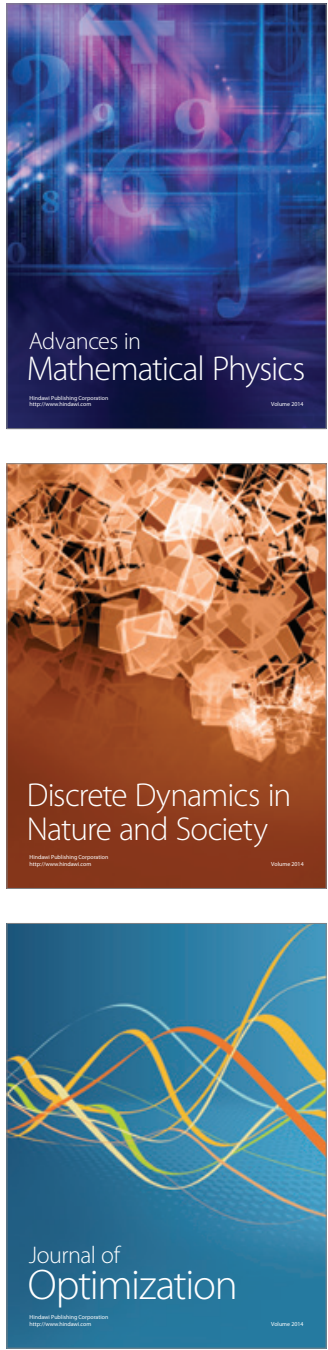